\title{
El crowdfunding llegó al Perú, ¿para quedarse?
}

Ricardo Mercado Espinosa

Abogado por la Universidad de Lima. Miembro Asociado de ADV Editores - Revista ADVOCATUS.

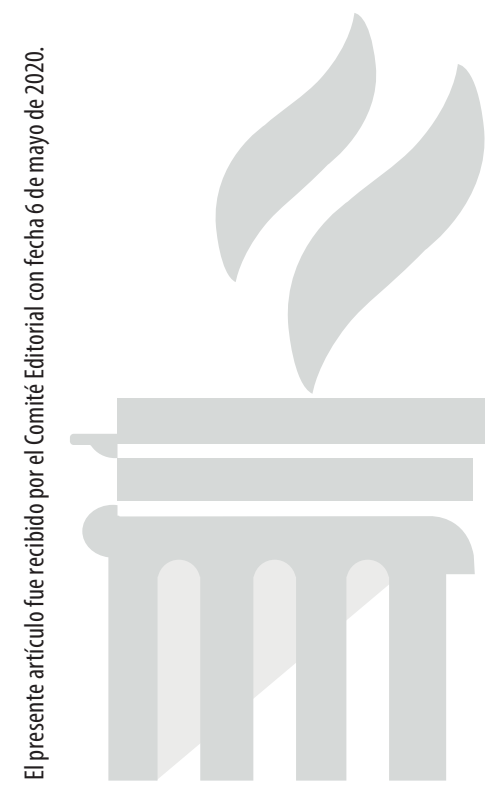

SUMARIO:

I. Introducción.

II. Crowdfunding: antecedentes y conceptos.

III. Modalidades.

IV. Principales ventajas y desventajas.

V. Riesgos típicos.

VI. Regulación.

VII. ¿Por qué es atractivo el crowdfunding en países como el Perú?

VIII. Desafíos para los actores del crowdfunding.

IX. Conclusiones. 


\title{
RESUMEN:
}

El presente artículo busca describir brevemente el fenómeno del crowdfunding o financiamiento participativo, como un mecanismo de financiamiento alternativo para emprendedores que, en su mayoría, tienen barreras para acceder a financiamientos en el sistema financiero tradicional. Asimismo, tiene como finalidad poner sobre la mesa los principales desafíos que tienen todos los actores del crowdfunding para que este mecanismo permanezca en el tiempo y ayude al desarrollo de la economía peruana.

Palabras clave: crowdfunding, financiamiento participativo, emprendimiento, desafíos.

\begin{abstract}
:
This article seeks to briefly describe the phenomenon of crowdfunding or participatory financing as an alternative financing mechanism for entrepreneurs who, for the most part, have barriers to access to funding in the traditional financial system. It also aims show the main challenges that actors of crowdfunding have so that this mechanism stays in time and helps the development of Peruvian Economy.

Keywords: crowdfunding, participatory financing, entrepreneurship, challenges.
\end{abstract}

\section{INTRODUCCIÓN}

En junio de 2016 William perdió a Edward, su hermano, debido a un accidente automovilístico causado por una negligente conductora. Luego de dicha pérdida, William se unió con una asociación que tiene como fin ayudar y orientar a las víctimas o personas afectadas por accidentes de autos, y juntos buscan crear conciencia en la sociedad a través de campañas de seguridad vial. Sin embargo, William y la asociación necesitan fondos para implementar y publicitar las mismas, por lo que decidieron lanzar una campaña en la página web "gofundme. $\mathrm{com}^{\prime \prime}$, página de crowdfunding, con la finalidad de levantar el capital suficiente para ejecutar sus planes. ${ }^{2}$

Así como William, actualmente muchas personas naturales y jurídicas buscan mecanismos de financiamiento alternativos como el crowdfunding para concretar sus proyectos, ya sea porque consideran al crowdfunding un mecanismo de financiamiento que se adecúa mejor a sus necesidades, porque este tipo de mecanismos pueden publicitar de manera más adecuada su proyecto o debido a que no califican en el mercado financiero para un préstamo tradicional por el monto que requieren. De cualquier manera, el crowdfunding se ha convertido en uno de los mecanismos de financiamiento de moda para muchos emprendedores y personas que buscan ayuda económica. Es por ello que muchos países han optado por emitir normas y/o lineamientos específicos para regular este tipo de mecanismos de financiamiento. En esa misma línea, el pasado 23 de enero de 2020 el poder ejecutivo del Perú emitió el Decreto de Urgencia 023-2020, Decreto de Urgencia que promueve el financiamiento de las MIPYME, Emprendimientos y Startups —en adelante, el "Decreto de Urgencia" - que tiene como objetivo implementar medidas que permitan inyectar de liquidez a las MIPYME - micro, pequeña y mediana empresa - a través de, entre otros mecanismos, el crowdfunding o financiamiento participativo.

Teniendo en cuenta ello, el presente artículo tiene como finalidad ahondar en el fenómeno del crowdfunding, la importancia que puede llegar a tener este mecanismo de financiamiento en un país como el Perú y esbozar los prin-

1. La página web https://www.gofundme.com es una de las principales páginas de crowdfunding en el mundo.

2. El caso antes señalado ha sido recuperado de la siguiente página web: https://www.gofundme.com, específicamente del siguiente enlance: https://www.gofundme.com/f/stop-accidentesroma-2020. 
cipales desafíos que tienen todos los actores relacionados para lograr que el crowdfunding funcione de manera óptima en el país como lo viene haciendo en otros países, tanto desarrollados como en vías de desarrollo.

\section{CROWDFUNDING: ANTECEDENTES Y CONCEPTO}

El crowdfunding o financiamiento participativo es, básicamente, una forma de financiamiento en virtud del cual una persona publicita un proyecto de su autoría - que puede ser un emprendimiento de negocio o no- en una cierta plataforma - hoy en día, básicamente a través de las plataformas electrónicas - solicitando a una masa de inversionistas que le otorguen el financiamiento requerido para el desarrollo de su proyecto.

Así, el crowdfunding tiene 3 elementos principales:

a) La masa de inversionistas que son quienes financiarán, a través de pequeños montos de dinero otorgados por cada inversionista, los proyectos presentados en una determinada plataforma. Hoy en día muchas personas optan por realizar inversiones a través de este tipo de alternativas debido a una serie de razones. Las principales son las siguientes:

(i) Es accesible a cualquier persona. Si bien en todas las plataformas de crowdfunding es necesario un previo registro para invertir en los proyectos publicados en dichos portales y hay que cumplir la regulación propia del portal de crowdfunding, el proceso de registro es bastante sencillo y suele ser gratuito, en comparación con los procedimientos para acceder a otros mercados más sofisticados.

(ii) Bajos costos de transacción. Los costos de transacción para un inversionista de invertir en un proyecto en una plataforma de crowdfunding son bastante más bajos que realizar una inversión en el mercado financiero tradicional. (iii) Diversas oportunidades de inversión. A través de las plataformas de crowdfunding, un inversionista puede obtener información y luego realizar inversiones sobre una serie de proyectos publicados, tanto en el mercado local como en mercados internacionales.

(iv) Pronto acceso a nuevos productos. En el tipo de crowdfunding-premio, el inversionista obtendrá, antes que cualquier otro consumidor no inversionista, el producto relacionado con el proyecto financiado antes que dicho producto salga al mercado.

(v) Filantropía. En el crowdfunding-donación, el inversionista tiene la oportunidad de realizar donaciones a una serie de proyectos que buscan el bienestar de la comunidad o de personas o grupos específicos, tal como lo veremos más adelante.

b) La plataforma, es el elemento principal de este modelo de financiamiento, dado que su principal función es servir como conector de los inversionistas con los usuarios o creativos que buscan financiamiento. Hoy en día, las plataformas son electrónicas y el desarrollo de la tecnología ha hecho posible que las mismas generen interacciones entre una gran cantidad de personas, que sean más dinámicas y a costos muy bajos. Estas plataformas cuentan con sus propias políticas, lineamientos y términos y condiciones a efectos de que la comunicación entre inversionistas y creadores o creativos sea óptima tanto en el momento previo de la inversión, como en el post- inversión, una vez que el proyecto financiado se esté ejecutando.

c) Los creadores o emprendedores son las personas que solicitan un monto determinado a través de la plataforma, a efectos de desarrollar o materializar proyectos propios. Los creadores usualmente optan por financiarse a través de este mecanismo debido, principalmente, a los siguientes motivos:

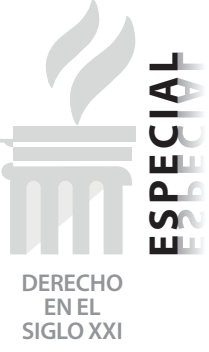

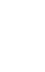


(i) No califican como sujetos de crédito. Una serie de personas naturales y jurídicas, por el contexto económico y su propio contexto personal no califican como sujetos de crédito bajo el modelo de financiamiento tradicional, por lo que buscan financiar sus proyectos a través de alternativas distintas, como es crowdfunding.

(ii) Al igual que para los inversionistas, los costos del financiamiento son más bajos. En comparación con la banca tradicional, financiarse a través de mecanismos como el financiamiento participativo son bastante más eficiente para determinadas empresas, ya que lo único que deberán pagar son las comisiones a la plataforma donde su proyecto ha sido publicado.

(iii) En los crowdfunding de inversión, la interacción del inversionista con el creador permite un flujo de comunicación y sugerencias respecto al producto que el creador lanzará al mercado que podría ser muy beneficioso para el creador.

Si bien en estos días este mecanismo de financiamiento alternativo se encuentra en auge o de moda, no es una creación nueva de las masas, sino bastante antigua. Sin embargo, en estos últimos tiempos ha adquirido gran importancia e interés de los diversos agentes económicos de la sociedad principalmente porque el entorno económico negativo en muchos países luego de las últimas crisis económicas generó que muchos emprendedores no puedan acceder a financiamientos otorgados por la banca tradicional y busquen diferentes y nuevas alternativas para financiar sus proyectos. Sumado a ello, hoy en día la interacción entre las personas es mucho más dinámica por el constante cre- cimiento de las redes sociales y el internet, lo que hace que el crowdfunding sea mucho más eficiente e interesante para todos los agentes involucrados que hace varios años, cuando no existía ese dinamismo entre los usuarios o creadores y los inversionistas.

Teniendo en cuenta ello, para Rodríguez de las Heras $^{3}$ en el crowdfunding confluyen factores económicos, sociales y tecnológicos que, juntos, tratan de responder a los nuevos desafíos de un mercado de financiamiento que se encuentra atravesando una profunda crisis, junto con una sociedad que empieza a cuestionar la banca tradicional. Así las cosas, este nuevo tipo de mecanismo de financiamiento tecnológico nace como una respuesta social al contexto adverso señalado anteriormente, lo que genera que el crowdfunding tenga esa naturaleza colaborativa entre sus miembros.

\section{MODALIDADES}

De acuerdo a Rodríguez de las Heras ${ }^{4}$ y coincidente con la mayoría de los autores, el crowdfunding, por la naturaleza de la transacción que se lleva a cabo en cada uno, principalmente tiene cuatro modalidades:

a) Donación: esta modalidad se caracteriza porque el inversionista que otorga el financiamiento no recibe una retribución o retorno en relación con el dinero invertido, sino que es puramente filantropía. Este tipo o modalidad de crowdfunding es una de las primeras modalidades utilizadas y permite que proyectos sociales o individuales específicos sean publicitados y puedan acceder al financiamiento que esperan. Por ejemplo, dentro de esta modalidad se encuentra el caso de William, caso con el que inicié el presente artículo; sin embargo, existen una infinidad de ca-

3. Teresa Rodríguez de las Heras Ballell, «El Crowdfunding: una forma de financiación colectiva, colaborativa y participativa de proyectos», Revista Pensar en Derecho, n. 3 (2015): 103.

4. Ibíd., 108. 
sos de mayor trascendencia relacionados a esta categoría, dentro de los cuales se encuentran, por ejemplo, los casos médicos, es decir personas que necesitan ayuda económica para cubrir operaciones, tratamientos, medicamentos, o cualquier otro servicio o producto relacionado a una enfermedad que se encuentren atravesando; los casos académicos, personas que necesitan de financiamiento para terminar su carrera universitaria, maestría o doctorado; $y$, programas científicos o escuelas de ciencias que dejan de contar con el financiamiento público de ciertos países y se mantienen operando debido a mecanismo de financiamiento colaborativos como el crowdfunding.

Por ejemplo, esta modalidad es muy usada en Canadá 5 para financiar, justamente, casos relacionados al cuidado de la salud y al sector educativo. Así, el crowdfunding-donación, sirve muchas veces para reemplazar de alguna manera al Estado en la protección de la salud y el acceso a la educación de calidad de muchas personas que no tienen los recursos necesarios, ya sea porque no cuentan con seguros médicos, porque los seguros médicos con los que cuentan no les cubren las operaciones o medicamentos que necesitan por determinadas enfermedades o porque las maestrías o programas educativos a las que aspiran son muy costosas para que un estudiante las pague.

Asimismo, en la actualidad esta modalidad también ha servido para financiar proyectos científicos y académicos de organizaciones que antes tenían el soporte público, pero que, por las diferentes crisis, perdieron dicho apoyo y, gracias a financiamientos colaborativos como el crowdfunding, siguen subsistiendo hasta el día de hoy.

b) Premio: esta modalidad se caracteriza porque los aportes de los inversionistas son recompensados o retribuidos por beneficios otorgados por el emprendedor o creador relacionado. Cabe señalar que estos beneficios o recompensas no deben ser económicos, sino vinculados al proyecto financiado. Usualmente la recompensa otorgada es el mismo producto que el emprendedor desarrollará con el financiamiento otorgado, solo que el inversionista obtendrá dicho producto antes que cualquier otro consumidor dentro del mercado.

Una de las ventajas de esta modalidad ${ }^{6}$, es que permite que los emprendedores que publican sus proyectos, a través de la interacción con los posibles inversionistas, obtengan una retroalimentación sobre los productos relacionados con dicho proyecto que podría ser muy provechosa para el emprendedor.

Asimismo, esta modalidad permite que los inversionistas que apoyen económicamente al proyecto publiciten el producto relacionado y se generen expectativas sobre dicho producto antes de que salga al mercado.

Un claro ejemplo que involucra las características antes descritas de esta modalidad es el caso de la start-up Pebble7, que en el 2012 llegó a recaudar 10.3 millones de dólares mediante la página Kickstarter, cuando solo

5. Martin Lukk, Erik Schneiderhan y Joanne Soares. «Worthy? Crowdfunding the Canadian Health Care and Education Sectors», Canadian Review of Sociology 55 (2018), 405.

6. Cabe señalar que la característica descrita no es exclusiva de esta modalidad, ya que también puede ser aplicada a las modalidades de préstamo y aporte de capital; sin embargo, la retroalimentación señalada es más usual en esta modalidad.

7. La noticia señalada ha sido recuperada de la siguiente página web: https://www.technologyreview.es/s/5948/ pebble-logra-la-cuarta-campana-mas-exitosa-en-la-historia-de-kickstarter. 
aspiraba a recaudar 100,000.00 dólares. Dicha campaña generó una retroalimentación entre los actores de la plataforma que la empresa emprendedora aprovechó, así como la gran recaudación realizada y la publicidad de los inversionistas generaron mucha expectativa en el público en general por la salida a la venta de la primera versión de sus relojes inteligentes.

c) Préstamo: esta modalidad tiene como finalidad otorgar préstamos a favor de los emprendedores a cambio del pago de intereses. Estos préstamos pueden tener o no tener garantías, dependiendo del emprendedor que solicite el financiamiento. Al respecto, cabe señalar que, esta modalidad puede ser estructurada de diversas formas, dependiendo de los términos y condiciones de cada plataforma y de la función que tenga la misma a lo largo de la vida del préstamo.

Por un lado, puede estructurarse de tal forma de que la plataforma solo sirva de interlocutor entre los emprendedores que buscan financiarse y los inversionistas. Por otro lado, es bastante más usual que la plataforma tenga una función más activa a lo largo del financiamiento. Estas funciones adicionales pueden ser, gestionar la estructuración del financiamiento, encargarse de recaudar el mismo y luego transferirlo al emprendedor, así como recaudar el pago del préstamo y luego transferirlo a cada inversionista, e inclusive, encargarse de la cobranza del financiamiento, en caso de un incumplimiento o un proceso de insolvencia por parte del emprendedor.

Una de las principales ventajas de esta modalidad es que, al no ser una actividad regulada como el mercado financiero tradicional o, en muchos países, tener una regulación muy light, los costos de transacción asociados son bastante bajos $y$, por lo tanto, es una alternativa más accesible para los emprendedores.

Así, los creativos o emprendedores que ne- cesitan capital de trabajo para impulsar sus proyectos, pero que no pueden acceder a la banca tradicional por diversos factores, ya sea porque su emprendimiento no calificó para la entidad del sistema financiero o porque no cuentan con activos para otorgarlos en garantía, buscan financiarse a través de este tipo de mecanismos.

Es pertinente recalcar que, teniendo en cuenta las características de esta modalidad, es una de las que ha crecido más en los últimos tiempos en países desarrollados y en vías de desarrollo.

d) Aporte de capital o equity crowdfunding: en virtud de esta modalidad, los inversionistas que otorgan el financiamiento reciben en calidad de contraprestación una participación en el capital de la persona jurídica emprendedora que buscó el financiamiento. De acuerdo a las diferentes plataformas que existen al día de hoy, esta modalidad se puede estructurar de dos maneras respecto a la contraprestación que recibirán los inversionistas. En la primera de ellas, los inversionistas pueden recibir royalties o utilidades futuras sin que lleguen a ser accionistas o socios de la emprendedora. En la segunda, los inversionistas, como contraprestación, reciben acciones o participaciones en el patrimonio de la persona jurídica emprendedora con todos los derechos políticos y económicos que ello conlleva.

Cabe señalar que la última "sub-modalidad" descrita implica una serie de riesgos que las partes o, en este caso, el emprendedor, debe regular a efectos de que tales riesgos no perjudiquen el proyecto que será financiado. Uno de los principales riesgos es que pueden existir nuevos inversionistas que sean complicados de manejar para el emprendedor $y$, como consecuencia de ello, el desarrollo del proyecto se vea atrasado o con problemas futuros.

Por último, usualmente bajo esta modalidad los emprendedores buscan un fi- 
nanciamiento a largo plazo y no solo una necesidad económica momentánea. Por el otro lado, los inversionistas no solo buscan recuperar la inversión en un periodo determinado de tiempo -como ocurre en la modalidad de préstamo-, sino que realizan una apuesta a largo plazo en el proyecto que desarrollará el emprendedor esperando una mayor ganancia a lo largo del tiempo.

Es pertinente indicar que usualmente las dos primeras modalidades señaladas no cuentan con regulaciones específicas debido a la naturaleza de las transacciones que se llevan a cabo dentro de dichas modalidades y las motivaciones con las que cuentan los inversionistas para entregar el financiamiento. Por otro lado, en muchos países es usual que se opte por regular las dos últimas modalidades, debido a que, por la naturaleza de las operaciones que conllevan, en el que existe un inversionista que coloca su dinero ahorro público - en una modalidad alternativa distinta al mercado tradicional y que tiene expectativas de un retorno esperado, es necesario establecer un régimen de protección a los inversionistas, junto con una serie de principios entorno a dicha protección. En esa misma línea, el Estado peruano, a través del Decreto de Urgencia ha optado, de manera expresa, por únicamente regular a las dos últimas modalidades de crowdfunding señaladas en la presente sección. ${ }^{8}$

\section{PRINCIPALES VENTAJAS Y DESVENTAJAS}

Tal como lo hemos observado a lo largo del presente artículo, el crowdfunding es un mecanismo alternativo de financiamiento que, tal como lo conocemos hoy en día, es relativamente nuevo, por lo que cada modalidad de crowdfunding cuenta con una serie de ventajas en relación al mercado financiero tradicional, pero también con varias desventajas que debemos tener en consideración.

En ese sentido, a mi criterio, las principales ventajas son las siguientes:

a) Una de sus principales ventajas es que es un nuevo canal de financiamiento que pueden aprovechar muchos emprendimientos que, teniendo buenas ideas y planes para desarrollar, no prosperaban y se quedaban fuera del mercado debido a que no calificaban para el otorgamiento de créditos por la banca tradicional. Lo anterior se puede deber a muchas cosas, pero las principales son, que muchas veces las sociedades que solicitaban el crédito no contaban con activos para otorgarlos en garantía, y/o que la banca tradicional no consideraba que los planes comerciales y financieros presentados eran idóneos para mantenerse en el tiempo y, como consecuencia de ello, para que puedan lograr el repago del préstamo.

b) Como ya lo hemos mencionado en un punto anterior del presente artículo, este mecanismo de financiamiento tiene costos de transacción bajos que, además, son muchas veces, determinados y predecibles de acuerdo a cada plataforma, usualmente los costos asociados serán las comisiones que deben ser pagadas a dichas plataforma- en comparación a los costos asociados al mercado tradicional que en muchos casos son bastante elevados ya que conllevan una serie de cadena de comisiones a ser pagadas por el prestatario, sin perjuicio de que los intereses a ser cobrados por el mercado tradicional serán bastante más altos debido al riesgo de incumplimiento que comprenden los emprendimientos en sus etapas iniciales.

c) La naturaleza colaborativa y social de este mecanismo de financiamiento y la interac-

8. En la sección VI hablaremos con mayor detalle del Decreto de Urgencia y los principios allí establecidos. 
ción entre las partes que supone pueden generar una retroalimentación importante sobre el proyecto que se busca financiar y desarrollar. Así, las sugerencias de los inversionistas, que a su vez buscan cuidar la inversión a realizar, pueden generar mejoras al plan inicial de los emprendedores.

d) En el caso del Perú y respecto de la modalidad de aporte de capital, otra de las grandes ventajas para recurrir a este mecanismo es que, como ya veremos en la sección VI siguiente, la normativa especial ha determinado que los emprendedores que quieran utilizar este mecanismo no deben cumplir con todos los requisitos establecidos por la Ley de Mercado de Valores -Decreto Legislativo 861- ya que este mecanismo no será considerado como una oferta pública en los términos establecidos en dicha ley, lo que automáticamente genera un gran incentivo para los emprendedores y plataformas electrónicas, teniendo en cuenta los tiempos y grado de sofisticación que implica cumplir con dichos requerimientos.

e) El uso de este mecanismo de financiamiento implica que las partes involucradas sean personas naturales o jurídicas formales, lo cual generará incentivos suficientes para que los emprendedores que busquen financiamiento en el mercado $-y$ que usualmente en ese estado del emprendimiento no son formales- se formalicen — sean personas naturales o jurídicas - y así puedan acceder a las plataformas de crowdfunding $\mathrm{y}$, dependiendo de su proyecto, acceder al financiamiento esperado.

Por otro lado, teniendo en consideración que el crecimiento de este novedoso mecanismo de financiamiento es reciente, también tiene una serie de desventajas que las partes involucradas deben tener en consideración. Las principales serían las siguientes:

a) Muchas veces en las fuentes de financiamiento tradicionales destinadas para emprendedores, ya sea en banca tradicional, venture capital o inversionistas ángeles, los emprendedores, además del financiamiento esperado, encuentran también un guía en cuanto al manejo y planeamiento del negocio, sus finanzas, contabilidad, etc. En el caso del crowdfunding, si bien existe una retroalimentación entre las partes, usualmente se limita al producto o productos relacionados al proyecto y no sobre el manejo financiero, estrategia de negocios y manejo contable que sí podría aportar otra modalidad de financiamiento.

b) Una de las principales desventajas que encontramos en este tipo de financiamiento para los emprendedores es que, por la naturaleza del crowdfunding, es necesario que el emprendedor publicite a través de las diferentes plataformas, el proyecto que requiere financiamiento, incluyendo detalles de los productos o servicios que ofrecerá al público, planes de negocio y otros detalles que podrían ser sensibles y podrían generar que otros emprendedores o empresas consolidadas tomen su iniciativa o ideas y las repliquen.

c) Otra de las desventajas, en este caso para los inversionistas, es que muchos emprendedores, si bien tienen ideas nuevas y un plan de negocio a corto plazo, muchos de ellos no cuentan con estrategias y planes de negocio a largo plazo por desconocimiento en diversas materias contables, financieras, de marketing, o de diversa índole, lo que podría generar que el proyecto no obtenga los flujos esperados $y$, como consecuencia de ello, que los inversionistas no obtengan las ganancias proyectadas sobre dicho proyecto.

d) Otra desventaja, únicamente aplicable a la modalidad de aporte de capital, es que, si las partes no regulan de manera óptima la relación jurídica que tendrán luego de haber efectuado la inversión, ya sea a través de un convenio de accionistas o cualquier otro documento similar, en calidad de accionistas de la sociedad que recibió el financiamiento, existe la posibilidad de que algún inversionista genere conflictos 
internos que devenguen en problemas en la administración de la compañía y que impacten en los planes de negocios planteados por el emprendedor.

\section{RIESGOS TÍPICOS}

Adicionalmente a las ventajas o desventajas que pueda tener el crowdfunding, también es necesario tener en consideración los riesgos típicos a los que se enfrentarán el Estado con el marco legal aplicable, los inversionistas y los emprendedores.

En ese sentido, los principales riesgos típicos que los agentes involucrados deben tener en cuenta son los siguientes:

a) Fraude: uno de los mayores riesgos relacionados al crowdfunding es que es un mecanismo que, por su naturaleza, puede generar que tanto las plataformas dedicadas a este tipo de mecanismo o los emprendedores engañen o defrauden a los inversionistas que, en su mayoría son personas naturales que buscan invertir su dinero sin tener conocimientos suficientes sobre el mercado financiero o tecnología como para darse cuenta si un proyecto o la plataforma donde se encuentra navegando tienen rasgos de ser fraudulentos.

Así, existen muchos casos en los que pseudo emprendedores publican proyectos muy atractivos falseando información contable, financiera o sobre el plan de negocios, que genera que muchos inversionistas inexpertos depositen sus ahorros en dichos proyectos fraudulentos. De igual manera, existen plataformas que buscan engañar a inversionistas publicando supuestos proyectos de supuestos emprendedores con información falsa. Al respecto, consideramos que la única manera de paliar este riesgo es a través de (a) un marco normativo claro, (b) la correcta difusión de información sobre las plataformas que se encuentran autorizadas por la autoridad competente y (c) creando una cultura alrededor de este mecanismo de financiamiento ${ }^{9}$ a efectos de que todos los actores ayuden a encontrar y eliminar proyectos falsos o plataformas no autorizadas que se encuentran en la red.

b) Lavado de activos: uno de los mayores riesgos relacionados a la implementación del crowdfunding en países como el Perú es que es muy sencillo que a través de plataformas como las del crowdfunding, muchas personas inescrupulosas se hagan pasar como inversionistas y/o emprendedores para lavar activos o financiar actividades ilícitas. Teniendo en cuenta ello, es absolutamente necesario la implementación de normas especiales para controlar el lavado de activos y el financiamiento del terrorismo en todos los agentes involucrados y que la supervisión sobre este punto sea dinámica por parte del regulador.

c) Incumplimiento o demora en cumplimiento del emprendedor: un riesgo bastante usual para los inversionistas es el incumplimiento del emprendedor de las obligaciones pactadas, dependiendo de la modalidad o la demora en su cumplimiento. Este riesgo es inherente al modelo de financiamiento y se produce por diversos factores entre los que se encuentran, la asimetría de información entre el emprendedor y el inversionista, la inexperiencia o incompetencia de los emprendedores en ciertas materias, que el proyecto no funcionara como se deseaba, que los emprendedores no realizaron planes aterrizados al mercado en el que se encuentran, lo que produce la demora en el cumplimiento de sus obligaciones.

Al ser éste un riesgo inherente al modelo de financiamiento, únicamente se podrá paliar a través de campañas de difusión de información y conferencias a todos los actores a efectos de crear inversionistas, em-

9. Ahondaremos más sobre este tema en la sección VIII siguiente. 
prendedores y plataformas cada vez más sofisticadas con la finalidad de que sean los propios agentes -emprendedores, plataformas e inversionistas- los filtros de buenos y malos emprendedores.

d) Seguridad cibernética: la seguridad cibernética es absolutamente necesaria para el correcto funcionamiento de este mecanismo de financiamiento, tanto para el emprendedor, que publica mucha información sensible de su negocio, como para el inversionista, que transferirá su dinero a través de la plataforma, y la plataforma relacionada. Si bien este punto es más un riesgo operacional para los actores involucrados que un riesgo inherente al mecanismo de financiamiento, es importante que las plataformas cuenten con seguridad cibernética certificada para otorgar confianza a los emprendedores y los inversionistas.

\section{REGULACIÓN}

Desde que inició la revolución del crowdfunding en muchos países del mundo, especialmente en Estados Unidos y ciertos países de Europa, también se inició un debate sobre si los estados deben o no regular este mecanismo de financiamiento. Al día de hoy, ese debate es menor y la opinión generalizada es que sí se debe regular el crowdfunding con la finalidad de paliar en la medida de lo posible los riesgos inherentes a dicho modelo de financiamiento. No obstante lo anterior, lo recomendable es que la regulación que emitan los estados no desnaturalice el modelo con sobre regulaciones que desincentiven el uso del crowdfunding por parte de todos los agentes involucrados.

En esa misma línea, con fecha 23 de enero de 2020 el Estado peruano publicó el Decreto de Urgencia, en virtud del cual establece, entre otras cosas, el marco jurídico aplicable para el financiamiento colectivo financiero o crowdfunding. Al respecto es necesario señalar que dicha norma únicamente es aplicable a las sociedades administradoras de plataformas orientadas a poner en contacto a personas naturales o jurídicas domiciliadas en el país - emprendedores, y que, en principio, desarrollen dicho proyecto íntegramente en el Perú - con una pluralidad de personas naturales o jurídicas o entes colectivos que buscan un retorno financiero -inversionistas-, con lo cual las únicas modalidades reguladas son la modalidad de préstamo y la modalidad de aportes de capital o emisión de deuda.

Asimismo, el Decreto de Urgencia ha establecido principios y disposiciones generales que luego deben ser complementadas con disposiciones que la Superintendencia del Mercado de Valores-SMV deberá emitir. Las principales disposiciones establecen (a) que la SMV será la autoridad que supervise a las sociedades administradoras de plataformas de financiamiento participativo financiero, (b) una serie de obligaciones para dichas sociedades con el fin de proteger a los futuros inversionistas, (c) una serie de prohibiciones para las sociedades administradoras con la finalidad de que presten un servicio idóneo a los inversionistas, y (d) la obligación de requerir información mínima de los futuros emprendedores y trasladar dicha información a los futuros inversionistas.

Por ello, a mi criterio, el Decreto de Urgencia ha regulado de manera general los puntos indispensables para iniciar el funcionamiento correcto del crowdfunding en el Perú; sin embargo, esperamos que las normas que emita la SMV vayan en esa misma línea y no solo regule de manera idónea el funcionamiento del crowdfunding y la protección a los inversionistas, sino también que pueda generar incentivos para que este mecanismo de financiamiento sea interesante para el mercado peruano.

\section{VII. ¿POR QUÉ ES ATRACTIVO EL CROWDFUN- DING EN PAÍSES COMO EL PERÚ?}

De acuerdo a estudios realizados por el Banco Mundial ${ }^{10}$, si bien el crowdfunding nació como un mecanismo de financiamiento en países de- 
sarrollados, puede servir como un instrumento para el desarrollo de economías de países en vías de desarrollo, como el Perú.

Para aterrizar la propuesta de esta entidad a la realidad del Perú es necesario tener en cuenta que al día de hoy existe una serie de emprendedores peruanos - no solo empresarios que recién se encuentren iniciando un proyecto, sino emprendedores medianamente consolidados con micros o pequeñas empresas funcionando-que tienen una serie de barreras para acceder a los canales de crédito tradicionales. Las principales barreras son que (a) los emprendedores muchas veces no cuentan con activos suficientes para garantizar el crédito solicitado, (b) o no cuentan con historial crediticio suficiente, lo que genera muchas veces que no sean sujetos de crédito, (c) muchos emprendedores no califican a la aprobación de los créditos porque no saben explicar o presentar la documentación requerida ante las entidades financieras, (d) las tasas en el mercado tradicional son muy altas para los emprendedores, (e) los costos asociados a los créditos muchas veces son muy elevados, entre otras causas.

Teniendo en consideración los problemas que aquejan a los emprendedores peruanos, un mecanismo de financiamiento con las características del crowdfunding es bastante atractivo para las personas que inician negocios o emprendimientos, micros y pequeñas empresas medianamente consolidadas y muchas iniciativas artísticas o culturales que requieren de financiamiento - sea corto, mediano o largo plazo-y así, a través de un mecanismo de financiamiento alternativo tecnológico, inyectar liquidez a la economía nacional y colaborar con el desarrollo de las microfinanzas en el Perú, sirviendo también como un mecanismo para luchar contra la pobreza y, como consecuencia, ayudar en el desarrollo del país.

Así lo han entendido una serie de agencias y/o entidades de fomento como el Banco Mundial o el Banco Interamericano de Desarrollo y otras entidades que están generando programas para fomentar el crowdfunding en países en vías de desarrollo, lo que podría ser aprovechado por el Perú, a efectos de consolidar en un corto plazo, el crowdfunding como un mecanismo de financiamiento alternativo.

\section{DESAFÍOS PARA LOS ACTORES DEL CROWDFUNDING}

Partiendo de la premisa de que, como se ha señalado en la sección anterior, el crowdfunding es un mecanismo de financiamiento que es atractivo para el desarrollo de las microfinanzas del Perú y que todos los agentes involucrados se encuentran de acuerdo con desarrollar el uso del crowdfunding en un corto o mediano plazo, es preciso señalar los desafíos a los que se enfrentan y los mecanismos que podrían utilizar como incentivos para que las personas hagan uso de este mecanismo. Así, en mi opinión el gran desafío para los agentes involucrados es la creación de una cultura del crowdfunding para que este mecanismo sea sostenible en el tiempo.

En ese sentido, el punto de partida para crear dicha cultura es que exista un marco legal óptimo que tenga como finalidad la protección del inversionista, pero sin que las obligaciones que imponga a los involucrados generen desincentivos para la utilización de este mecanismo de financiamiento, sino todo lo contrario, deberá incentivar a los emprendedores, sociedades administradores e inversionistas a la utilización de este mecanismo por su practicidad y eficiencia. Asimismo, dichas obligaciones deben ser pensadas para las personas naturales y jurídicas que utilizarán este mecanismo, por lo que no deberán ser obligaciones demasiado sofisticadas que generen costos excesivos en los emprendedores o sociedades administradoras de plataformas y que terminen asfixiando a los agentes involucrados en el mecanismo de financiamiento. Por otro lado, el marco legal debe tratar, en la medida de lo posible, de paliar o reducir los riesgos inherentes al modelo para evitar posibles fraudes, el lavado de activos y financiamiento del terrorismo a través de ella y evitar generar incentivos para incumplimientos por parte de los emprendedores.

Asimismo, será necesario que los agentes cuenten con una infraestructura adecuada para eliminar o reducir los riesgos operacionales más

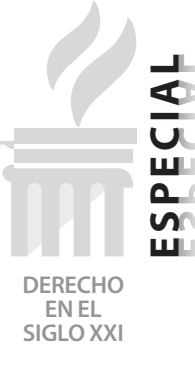


comunes. Así, las sociedades administradoras, como consecuencia de la aplicación del marco legal idóneo, deberán asegurar a los agentes involucrados que cuentan con una plataforma segura y que sus datos - muchos de ellos sensibles - se encuentran debidamente protegidos, que no podrán haber suplantaciones de los emprendedores que solicitan el financiamiento, intromisión de malas prácticas dentro de las plataformas, etc.

Por otro lado, como he mencionado en la sección VII anterior, existen muchas agencias de fomento y entidades multilaterales que han diseñado programas para el desarrollo del crowdfunding en países en vías de desarrollo, por lo que, sin duda, sería más que interesante que el Perú suscriba convenios con dichas agencias para tomar los modelos ya estructurados y aplicarlos en el país. Es pertinente señalar que dichas agencias están dispuestas, no solo a aplicar los programas diseñados, sino también en invertir dinero para incentivar el uso y sostenibilidad del crowdfunding.

Adicionalmente, el Ministerio de la Producción también podría ayudar en el desarrollo de este mecanismo de financiamiento, creando programas de incentivos. Por ejemplo, a través de concursos, podría elegir los mejores emprendimientos o los emprendimientos que cumplan con sus evaluaciones previas y financiar parcialmente dichos proyectos. Esa inversión parcial generaría confianza en los demás inversionistas $y$, son dichos inversionistas los que completa- rían la inversión total solicitada por los emprendedores.

Por último, es muy importante que el Ministerio de la Producción, la SMV y otras entidades gubernamentales, así como el sector privado, generen charlas, cursos o material educativo relacionado, para difundir el crowdfunding y qué conocimientos mínimos deben adquirir los agentes involucrados para su correcto funcionamiento, lo que publicitaría el mecanismo de financiamiento y, a su vez, generaría del mismo por parte del Estado y del sector privado.

\section{CONCLUSIONES}

Luego de ver qué es el crowdfunding, las modalidades que existen y sus principales características, considero que este mecanismo de financiamiento alternativo es una oportunidad muy interesante para el Perú para el impulso y desarrollo de emprendedores que antes tenían muchas barreras para acceder a financiamientos a través del mercado tradicional.

No obstante, para que el modelo funcione y sea sostenible en el tiempo, es necesaria la participación activa del Estado y todos los agentes involucrados para que, a través de los mecanismos propuestos en la sección anterior, se genere una cultura o ecosistema alrededor del mismo y se incentive su correcto funcionamiento, así como se eviten o reduzcan los riesgos inherentes al modelo, así como los riesgos operacionales relacionados.

Ajay K. Agrawal, Christian Catalini y Avi Goldfarb, «Some Simple Economics of Crowdfunding», Innovation Policy and the Economy, Working Paper n. ${ }^{\circ} 19133$ (2013).

ConnectAmericas, «El Crowdfunding llega a las PYMEs de América Latina y el Caribe», Conexión Intal, https://conexionintal.iadb.org/2018/01/04/crowdfunding-llega-las-pymes-america-latina-caribe/.

Elena Gil Moreno, «Nuevos activismos sociales en la era digital: de las masas al "crowd"», Política y Sociedad 54, n. ${ }^{\circ} 1$ (2016): 191-208.

Iván Abarca, «Desarrollo del Crowdfunding en Chile», Working Papers Central Bank of Chile n. 815 (2018).

Juan José 'Price y Felipe Mujica, Matchfunding: una alternativa de financiamiento para las industrias creativas (Banco Interamericano de Desarrollo, 2018).

Proyecto de Ley mediante el cual se propone la Ley que Regula el Régimen Jurídico de las Plataformas de Financiamiento Participativo - PFP, http://www.congreso.gob.pe/pley-2016-2021. 